\title{
Editorial: The Fiber Bundle
}

\author{
Alex Hansen ${ }^{1 *}$, Ferenc Kun ${ }^{2}$, Srutarshi Pradhan ${ }^{1}$ and Purusattam Ray ${ }^{3}$ \\ ${ }^{1}$ PoreLab and Department of Physics, Norwegian University of Science and Technology, Trondheim, Norway, ${ }^{2}$ Department of \\ Theoretical Physics, Doctoral School of Physics, Faculty of Science and Technology, University of Debrecen, Debrecen, Hungary, \\ ${ }^{3}$ The Institute of Mathematical Sciences, Chennai, India
}

Keywords: fiber bundle model, damage, fracture, statistical physics, materials science

\section{Editorial on the Research Topic}

\section{The Fiber Bundle}

A long time has passed since Fredrick Thomas Peirce, an Australian physicist working for the British Cotton Industry Research Association, published the paper Tensile tests for cotton yarns: "the weakest link" theorems on the strength of long and of composite specimens in 1926 [1]. In it, he describes a simplified model for failure of yarn where the variations of the strength in the yarn competes with increased load it carries when there is a failure. Here it is in modern language: Imagine a set of fibers of equal length clamped between two parallel stiff plates. The fibers all have the same elastic constant, so that when the plates are moved apart, all the fibers carry the same load, which is the total load on the plates divided by the number of fibers. Assume each fiber has its own strength, that is the maximum load it can carry before it fails irreversibly. As the fibers fail one by one, the load on the remaining fibers increases as there are fewer and fewer left to share the total load.

Such a model seems overly simple. It cannot possibly contribute to our understanding of fracture phenomena! The mathematical statistician Henry Daniels did not think so. He did his PhD on the strength of fiber bundles and in 1945 he published a seminal paper on his work that moves the model beyond the strength of yarn [2], reshaping it into a general model for failure processes. Up to the point when he published his paper, we find six papers referring to the Peirce paper [1]. ${ }^{1}$ In the decade that follows 1945, there are 35 references to the Peirce paper, but the titles have now changed. We find e.g., "The Fracture of Metals" [3]. ${ }^{2}$

It is in the seventies that the fiber bundle model really gains traction within the mechanics community [4]. Together with the statistics community, the subtleties of the model is gradually uncovered, while at the same time variants of it is being proposed, e.g., the Local Load Sharing Model [5]. ${ }^{3}$

The statistical physics community, those with a background in critical phenomena, had in the mid eighties began to discover that fracture in fact is a very interesting problem with properties that sometimes created a déjà $v u$ feeling among them [6]. This resulted in the creating of the Fuse Network Model [7]. This was network of fuses, e.g. a square lattice, where each link would be a fuse with a trip current drawn from some statistical distribution. What would happen when the current running through this network was increased? A lot of things would happen: there would be avalanches of fuses blowing simultaneously, there would be localization, i.e., the fuses that blew would be concentrated in some region, there would be instabilities where suddenly a crack of blown fuses would form zipping through the network. The problem with the fuse model, however, was that it was

Received: 15 October 2021

Accepted: 27 October 2021

Published: 18 November 2021

Citation:

Hansen A, Kun F, Pradhan S and Ray $P$

(2021) Editorial: The Fiber Bundle.

Front. Phys. 9:795803.

doi: 10.3389/fphy.2021.795803

${ }^{1}$ There is one exception among the six: "A Study of the Moisture in Soybeans" [11]. The reference to Peirce is for a property of cellulose fibers he mentions unrelated to the model.

${ }^{2}$ Peirce is lauded in Reference [3] for his paper [1] being the first discussion strength as extreme value problem, and not for his model.

${ }^{3}$ The original Peirce model is by now called the Global Load Sharing Model. 
almost impossible to approach it analytically-it was a model for the computer, and the computer only. The book by Herrmann and Roux from 1990 (and reprinted in 2014) covers the status of the field at that time [6].

In 1989 Sornette published a paper entitled Elasticity and Failure of a Set of Elements Loaded in Parallel [8]. This was the entrance of the fiber bundle model into the statistical physics community. It was the perfect model in some sense. It was almost as rich as the fuse network model, but it was at the same time simple enough to be analytically tractable to a considerable degree. In some sense, the fiber bundle model is gaining a status in the physics of fracture community similar to that of the Ising model in the critical phenomena community. Two reviews of the fiber bundle model have appeared in this community $[9,10]$. There are hundreds of papers, if not more, that have appeared over the last years in this field.

The aim of this Research Topic has been to display some of the richness of ideas and uses that the fiber bundle model has produced. The eleven papers it contains does indeed do that. Hopefully, the research papers among them spawn new work and the reviews are useful for those that considers entering this field.

We describe the papers in the order in which they have been published.

The first paper (Kjellstadli) concerns the burst distribution in the global load sharing fiber bundle model. Earlier work had determined it for large bursts, but here Kjellstadli finds it for small bursts. This is important as large bursts are rare compared to small bursts so that the statistics is much better for the latter.

The second paper (Capelli et al.) uses the fiber bundle model to investigate failure of layers of snow, failures that can lead to avalanches.

Paper three (Domanski) implements the local load sharing fiber bundle model on a small-world network. The avalanches that are generated in such networks may be very relevant for other systems that has an underlying small-world structure, such as the financial markets.

Paper four (Hansen) is a review of the extreme value distributions, which are central for the fiber bundle model. Rather than approaching the subject in the traditional way, the entire review is built around the formula $\lim _{n \rightarrow \infty}(1+x / n)^{n}=\exp (x)$.

Paper five (Chakrabarti et al.) is a review of how the dynamics in the fiber bundle model generates cooperativity. The failure dynamics has been demonstrated through recursion relations and their fixed-point solutions. In addition, the authors analyze the noise-induced failure dynamics through theory, simulation, and

\section{REFERENCES}

1. Peirce FT. 32-X.-Tensile Tests for Cotton Yarns v.-"The Weakest Link" Theorems on the Strength of Long and of Composite Specimens. J Textile Inst Trans (1926) 17:T355-T368. doi:10.1080/ 19447027.1926.10599953

2. Daniels HE. The Statistical Theory of the Strength of Bundles of Threads. I. Proc R Soc Lond A (1945) 183:405-35. doi:10.1098/rspa.1945.0011

3. Petch NJ. The Fracture of Metals. Prog Metal Phys (1954) 5:1-52. doi:10.1016/ 0502-8205(54)90003-9 real data (Earthquake catalog) analysis discussing also the emergence of self-organizing mechanism in the model.

Paper six (Halász et al.) generalizes the fiber bundle model in order to describe the mechanical response of systems which undergo a sequence of stick-slip cycles like granular packings, biological materials, or tectonic plates. With analytic calculations and computer simulations the authors explore the capabilities of the approach.

Paper seven (Arango-Restrepo et al.) introduces heat exchange with the surroundings and thereby place the fiber bundle model in a thermodynamical context. This is important for the use of the fiber bundle model for e.g. polymeric systems.

Paper eight (Roy and Biswas) considers the energy avalanche statistics in the local load sharing fiber bundle model. A nonlinear relation of the avalanche size and the released elastic energy is established and a scale free energy distribution is revealed with a high degree of robustness.

Paper nine (Engelbrecht-Wiggans and Phoenix) places the fibers in an elastically responding matrix in order to model creep rupture in fiber-reinforced composites.

Paper ten (Dębski et al.) tests out a prediction based on the fiber bundle model for when catastrophic failure of a loaded structure is imminent. The authors use a discrete element system to demonstrate that indeed the fiber bundle model prediction holds.

Paper eleven (Roy) constructs the phase diagram of the fiber bundle model in the three-dimensional parameter space of threshold disorder, range of load sharing, and system size. He explores the complex phase structure of the model and characterizes the emerging failure modes.

\section{AUTHOR CONTRIBUTIONS}

All authors have made a substantial, direct and intellectual contribution to the work, and approved it for publication.

\section{FUNDING}

This work was supported by the Research Council of Norway through its Centres of Excellence funding scheme, project number 262644 and it was supported by the National Research, Development and Innovation Fund of Hungary, financed under the 2020-4.1.1-TKP2020 funding scheme Project no. TKP2020-NKA-04 and K 119967.

4. Harlow DG, Phoenix SL. The Chain-Of-Bundles Probability Model for the Strength of Fibrous Materials I: Analysis and Conjectures. J Compos Mater (1978) 12:195-214. doi:10.1177/002199837801200207

5. Harlow DG, Phoenix SL. Approximations for the Strength Distribution and Size Effect in an Idealized Lattice Model of Material Breakdown. J Mech Phys Sol (1991) 39:173-200. doi:10.1016/0022-5096(91)90002-6

6. Herrmann HJ, Roux S. Statistical Models for the Fracture of Disordered media. ISBN 0444-88551x. Amsterdam: Elsevier (2014).

7. de Arcangelis L, Redner S, Herrmann HJ. A Random Fuse Model for Breaking Processes. J Phyique Lett (1985) 46:585-90. doi:10.1051/jphyslet: 019850046013058500 
8. Sornette D. Elasticity and Failure of a Set of Elements Loaded in Parallel. J Phys A: Math Gen (1989) 22:L243-L250. doi:10.1088/0305-4470/22/ $6 / 010$

9. Pradhan S, Hansen A, Chakrabarti BK. Failure Processes in Elastic Fiber Bundles. Rev Mod Phys (2010) 82:499-555. doi:10.1103/RevModPhys.82.499

10. Hansen A, Hemmer PC, Pradhan S. The Fiber Bundle Model: Modeling Failure in Materials. ISBN: 978-3-527-41214-3. Weinheim: Wiley-VCH (2015).

11. Beckel AC, Earle FR. Study of Moisture in Soybeans. Ind Eng Chem Anal Ed (1941) 13:40-3. doi:10.1021/i560089a013

Conflict of Interest: The authors declare that the research was conducted in the absence of any commercial or financial relationships that could be construed as a potential conflict of interest.
Publisher's Note: All claims expressed in this article are solely those of the authors and do not necessarily represent those of their affiliated organizations, or those of the publisher, the editors and the reviewers. Any product that may be evaluated in this article, or claim that may be made by its manufacturer, is not guaranteed or endorsed by the publisher.

Copyright $\odot 2021$ Hansen, Kun, Pradhan and Ray. This is an open-access article distributed under the terms of the Creative Commons Attribution License (CC BY).

The use, distribution or reproduction in other forums is permitted, provided the original author(s) and the copyright owner(s) are credited and that the original publication in this journal is cited, in accordance with accepted academic practice. No use, distribution or reproduction is permitted which does not comply with these terms. 